\title{
ALTERNATIVE METHODOLOGY FOR ASSESSING PART-THROUGH-WALL CRACKS IN CARBON STEEL BENDS REMOVED FROM POINT LEPREAU GENERATING STATION
}

\author{
Xinjian Duan ${ }^{1}$, Michael J. Kozluk ${ }^{2}$, Tracy Gendron ${ }^{3}$, John Slade ${ }^{4}$ \\ ${ }^{1}$ Senior Stress Analyst, Reactor Engineering, AECL, Mississauga, ON, Canada (duanx@aecl.ca) \\ ${ }^{2}$ Principal Engineer, Reactor Engineering, AECL, Mississauga, ON, Canada \\ ${ }^{3}$ Manager-HTS Materials Integrity, AECL, Chalk River, ON, Canada \\ ${ }^{4}$ Senior Technical Advisor, Point Lepreau Generating Station, Lepreau, NB, Canada
}

\begin{abstract}
New Brunswick Power Nuclear has been successfully managing intergranular, axial cracking of carbon steel feeder piping, that is also experiencing thinning, in the Point Lepreau Generating Station, primarily by an aggressive program of inspection, repair and testing of ex-service material. For the past three maintenance outages, a probabilistic safety evaluation (PSE) has been used to demonstrate that annual inspection of the highest risk locations maintains the nuclear safety risk from cracking at an acceptably low level. The PSE makes use of the Failure Assessment Diagram (FAD) model to predict the failure of part-through-wall cracks. Burst testing of two ex-service pipe sections with part-through-wall cracks has shown that this FAD model significantly under predicts the failure pressure from the component tests. Use of this FAD model introduces undesirable conservatism into PSE assessments that are used to optimize feeder piping inspection and maintenance plans. This paper describes an alternative finite element approach, which could be used to provide more representative structural models for use in PSE assessments. This alternative approach employs the elasto-plastic large strain finite element formulation; uses representative material properties; considers the spatial microstructural distribution; accounts for the effect of work hardening rate; models all deformation processes, i.e., uniform deformation, localized necking, and failure imitation and propagation. Excellent pre-test prediction was shown for the burst-pressure test in 2006 . Although cold-worked feeder bends have reduced fracture toughness compared to the parent straight pipe, post-test metallurgical examinations showed that failure at the test temperature $\left(90^{\circ} \mathrm{C}\right)$ is a ductile process, which is consistent with the alternative finite element approach. These finite element simulations have also shown that the cold-worked material with $35 \%$ pre-strain has greater load-carrying capacity (i.e., larger failure pressure) than the parent straight pipe.
\end{abstract}

\section{INTRODUCTION}

New Brunswick Power Nuclear (NBPN) Corporation has been managing some serious degradations in SA 106 Grade B carbon steel reactor coolant piping in the Point Lepreau Generating Station (PLGS) since 1996 [1]. Life limiting wall thinning from flow accelerated corrosion, and axial intergranular cracking that initiated from both inside and outside surfaces [2] has led to the premature replacement of seventeen outlet tight radius cold bends, located immediately downstream of the reactor core.

The outside surface cracking has been the most challenging to manage for the following reasons:

- A large percentage of removed tight radius bends were found to contain incipient outside surface cracks $(50-200 \mu \mathrm{m}$ deep), suggesting that a large population of in-service bends may be susceptible to deeper cracking;

- Cracks have been observed to develop from an un-detectable size to $\sim 75 \%$ through-wall within one year of operation;

- $\quad$ Outside surface cracks occur at the bend extradoses where the margins on crack stability are lowest because of the lower wall thickness, reduced fracture toughness, and the potential to develop longer partial-through-wall cracks.

In response to these and other challenges, NBPN adopted a probabilistic approach to manage feeder cracking, supported by burst-pressure tests of ex-service bends with part-through-wall cracks. A Probabilistic Safety Evaluations (PSE) is currently used to demonstrate that annual inspection of the highest risk locations maintains the nuclear safety risk from cracking at an acceptably low level [3]. Crack stability models that are used to calculate the probability of pipe rupture of axial, partial through-wall and through-wall cracks are an important component of the PSE.

The FAD model of Section XI of the ASME B\&PV Code has been widely used to predict the failure of part-throughwall cracks in the PSE assessments. This model is not ideal for this purpose of failure avoidance and is believed to introduce penalizing conservatism into PSE assessments, for several reasons:

- The FAD model was developed to identify conditions where rupture will not occur, with a high degree of confidence, not to provide best-estimate predictions; 
- $\quad$ Because of the small size of feeder pipes (2" and 2.5"), it is necessary to extrapolate the use of the Section XI FAD model to pipe sizes less than NPS 4 (Nominal Pipe Size).

- The FAD model significantly under-predicted the failure pressures of the ex-service feeders when flaw proximity rules of Section XI are applied.

This paper describes an alternative Heterogeneous Finite Element Model (HFEM), which is considered to provide more realistic prediction of feeder behavior. The key elements of the HFEM include:

- Employing an elasto-plastic large strain finite element formulation;

- $\quad$ Using realistic material properties and considering the spatial microstructural distribution;

- Accounting for the effect of work hardening rate; and

- Modeling all deformation processes: uniform deformation, localized necking, and failure initiation and propagation.

This HFEM has been extensively validated against experimental measurements for both steels and aluminum alloys under all deformation states: plane stress, plane strain, and 3-D [4]. This HFEM has also been successfully applied to assess the structural integrity of degraded steam generator tubes with a variety of defects, such as pitting, uniform thinning, and axial cracks [5].

\section{BURST-PRESSURE TESTS OF FEEDER BENDS WITH DEEP PART-THROUGH-WALL CRACKS}

NBPN has used burst-pressure testing of removed feeder bends with axial surface cracks to provide a strong technical basis for its PSE assessments. The testing results are not only used to validate the crack stability model, but also to improve the Probability of Detection (PoD) and the crack growth rate model. Two feeder sections with extrados crack-like indications were tested to failure at $90^{\circ} \mathrm{C}$, feeder D14 in 2005 and feeder H12 in 2006 . Figure 1 shows the crack field revealed using magnetic particle inspection prior to testing. The image shows a colony of discontinuous axial surface indications located at the centre of the extrados of the second bend. Compared with the crack field in D14, cracks in H12 are longer (100 mm vs. $55 \mathrm{~mm}$ ) and deeper (73\%tw vs. 59\%tw).

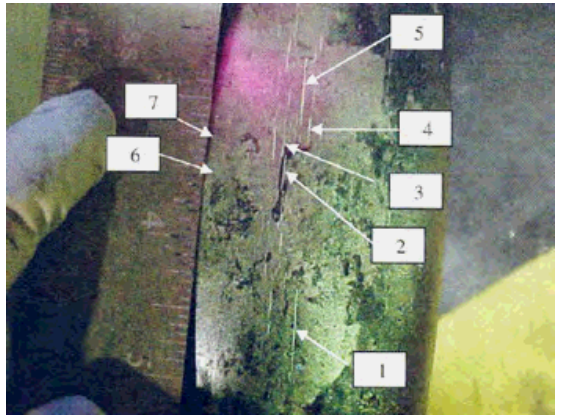

Feeder D14 Tested in 2005

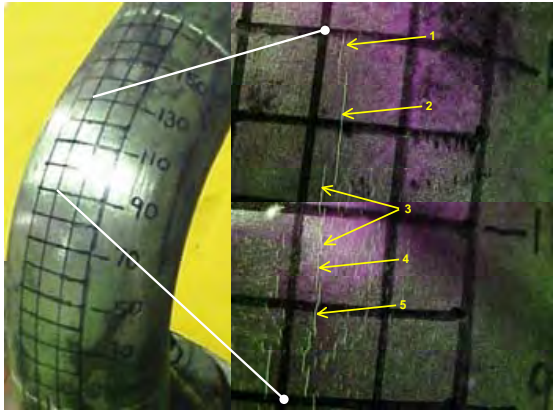

Feeder H12 Tested in 2006

Figure 1: Crack Field Obtained by Magnetic Particle Inspection.
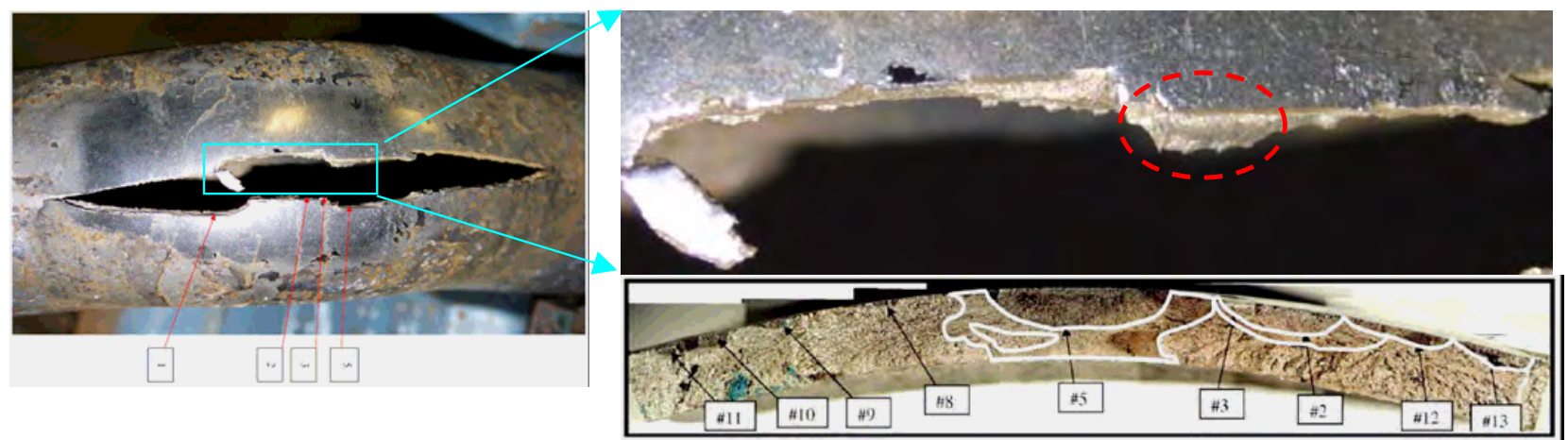

Figure 2: Ductile Controlled Failure Modes

Figure 2 shows the region of failure in the D14 bend after burst testing. White lines on the bottom photograph of the fracture surface identify ductile crack extensions. Of particular interest is the existence of shear slip at the bottom of the 
major surface crack \#5. The formation of this shear slip is associated with local plastic instability of the remaining throughwall ligament that initiated the failure. In-depth discussion of this shear slip is given in Section 4.5.

\section{LIMITATIONS OF FAILURE ASSESSMENT DIAGRAM}

Subarticle IWA-3300 of Section XI of the ASME B\&PV Code [6] provides procedures for characterizing the size of detected flaws. If these procedures were applied to the indications of Figure 1, it would be necessary to characterize the flaw as having a length equal to the axial extent of the region of the crack field. Using these procedures, the flaws in feeders D14 and H12 would be characterized as having a total length of $40 \mathrm{~mm}$, and close to $100 \mathrm{~mm}$, respectively. However, the results of the burst-pressure tests (Figure 2) clearly show that the failure was associated with the plastic instability of the remaining through-wall ligament of the deepest of the indications (\#5 and \#2). Using the FAD for axial flaws in a straight pipe from Appendix H of Section XI, the ASME Code specified yield strength and ultimate strength, and the average fracture toughness from the compact toughness tests $\left(J_{I c}=18 \mathrm{~kJ} / \mathrm{m}^{2}\right)$ at $265^{\circ} \mathrm{C}$ the predicted failure pressure for 2005 test is $17 \mathrm{MPa}$. This failure pressure (17 $\mathrm{MPa}$ ) is less than $25 \%$ of the actual failure pressure.

Aside from the use of a straight-pipe analogy for the assessment of axial cracks in feeder bends, there are two major issues in the application of the FAD flaw model.

- IWB-3641(a) stipulates that the FAD is applicable for pipe NPS 4 and larger.

- IWB-3641(b) stipulates that the FAD is applicable for carbon steel pipes that have a specified minimum yield strength not greater than $280 \mathrm{MPa}$.

Although both conditions are violated for the cold-worked PLGS feeder bend, for the first limitation on pipe size, ASME Section XI subcommittees consider that there are no technical limitations to apply FAD to a pipe size down to 1" and in fact work is currently underway to remove this limit. For the second condition, the yield and ultimate tensile strengths for the extrados regions of the $2 \frac{1}{2} 2^{\prime \prime}$ NPS tight-radius feeder bends at the test temperature $\left(90^{\circ} \mathrm{C}\right)$ are estimated to be $627 \mathrm{MPa}$ and $707 \mathrm{MPa}$, respectively. However, the under prediction of the burst pressure for D14 (i.e. $17 \mathrm{MPa}$ ) confirms that it is more appropriate to use the actual material properties. In fact, this is the practice of other flaw evaluation procedures such as SINTAP and R6 [7]. When measured mechanical properties are used, the FAD model predicts a failure pressure of $28 \mathrm{MPa}$ with a $J_{I C}$ of $18 \mathrm{~kJ} / \mathrm{m}^{2}$ and an effective flaw length of $40 \mathrm{~mm}$. This failure pressure is 2.7 times of the normal operating pressure. Based upon the post-test metallurgical examinations for the 2005 test specimen in Figure 2, it is concluded that the failure initiated by ductile extension from the pre-existing surface cracks denoted as MPI indications \#5 and \#2, which are near the apex of the bend. The ductile region of \#5 is much larger than that of \#2. The initial ductile extension centering on indication \#5 was in the radial-axial plane. After this ductile extension, the remaining ligament failed by cleavage, creating a through-wall axial crack. Therefore, it is reasonable to assume that the effective crack length should be the length of indication \#5, i.e., $12.7 \mathrm{~mm}$. Using this value and the measured fracture toughness $J_{I c}$ of $18 \mathrm{~kJ} / \mathrm{m}^{2}$, the predicted failure pressure is $40.5 \mathrm{MPa}$. This failure pressure is still only $58 \%$ of the actual failure pressure.

The conservatism in the predicted failure pressure comes from several factors.

- The FAD was not intended to provide an accurate prediction of failure loads, it was developed to be conservative and is used as a break avoidance flaw model.

- Ductile extension is not taken into account because a $J_{r}$ resistance curve is not available for the cold-worked material.

- Fracture toughness measurements from side-grooved compact toughness, C(T), test specimens are conservative. This is attributed to the different constraint conditions between the $\mathrm{C}(\mathrm{T})$ specimen and the pipe component.

\section{HETEROGENEOUS FINITE ELEMENT METHOD (HFEM)}

In this section, the HFEM is described and used to simulate the 2006 burst-pressure test of feeder H12. The relative depth of the deepest indication is 73\%tw. It is noted that Section XI of the ASME B\&PV Code places an upper limit on the flaw depth of $75 \%$ tw. For flaw depth greater than this value, fracture mechanics methodologies are not applicable because the crack-tip plastic zone penetrates the remaining ligament. The failure is dominated by plastic collapse. The prediction from the FAD then depends on the accuracy of the limited solutions adopted by the FAD.

\subsection{Consideration of Microstructure}

Most commercially produced alloys have inhomogeneities presented at different length scales. Micro-scale and mesoscale refers to, for simplicity, microstructure with a size less than or greater than the grain size, respectively. At the microlength scale, the second phase particles are a major source of inhomogeneity. At the meso-scale, texture is a major source of inhomogeneity. The volume fraction of each texture component in a material can be measured using X-ray diffraction, neutron diffraction or electron back scattered diffraction (EBSD). The strength difference between the regions with average behavior (the so called "matrix") and the inhomogeneities can also be quantitatively calculated by the use of the Fourier 
transformation from measured EBSD data. The analysis is simplified; the inhomogeneity is treated phenomenologically by assigning regions that have a slightly higher or lower strength than the matrix. The matrix is assumed to have the average stress-strain response. The stress -strain curves for the "hard" and "soft" components are simply obtained by slightly shifting the stress-strain curve for the matrix up or down $\pm 3 \%$. Thus the work hardening rate for all three phases are the same. One of the three material properties was randomly assigned to each element. The volume fraction of each material in the finite element model is close to $33.3 \%$.

\subsection{Stress-Strain Curves}

A limited amount of fracture toughness and tension test material characterizations for feeder bends were performed because of cracking concerns, and for the straight pipes for the purposes of comparison. Both archive and ex-service materials have been characterized. Because of the limited volume of material available for testing from the bend region, the use of sub-size specimens is required. The tensile specimen geometry was developed and qualified in consideration of the ASTM standards E21 and E8. The testing was performed at room temperature, $265^{\circ} \mathrm{C}$, and $310^{\circ} \mathrm{C}$ at a strain rate of 0.0001 per second. Figure 3 shows the stress-strain curves used in the simulations. Material characterizations also show for SA-106 Grade B steel, the anisotropy in tensile properties is insignificant. However, there are substantial differences in the measured fracture toughness.

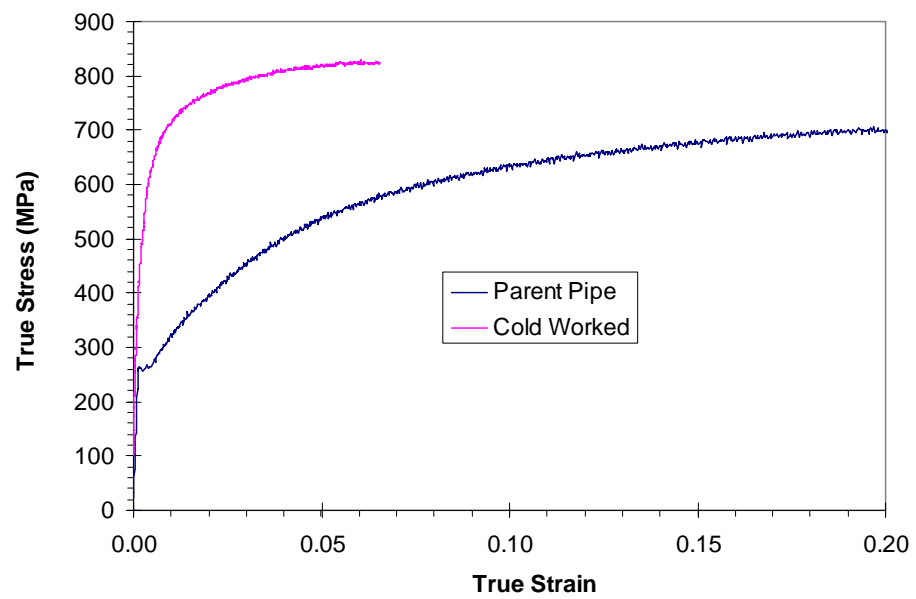

Figure 3: Stress-Strain Curves

\subsection{Ductile Failure Criterion}

The Rice and Tracey fracture criterion [8] is used to indicate the initiation of a crack and its subsequent propagation. This can be expressed as:

$$
\int_{0}^{\bar{\varepsilon}_{f}} e^{1.5 \sigma_{m} / \bar{\sigma}} d \bar{\varepsilon}=C
$$

where $\bar{\varepsilon}_{f}$ is the equivalent strain at fracture initiation, while $\sigma_{m}$ and $\bar{\sigma}$ are the hydrostatic stress and the equivalent stress, respectively. $C$ is failure parameter, to be determined by matching the predicted failure pressure with the measured failure pressure for a single case.

It is worth pointing out that the R6 procedure includes a similar ductile failure criterion [10]. Even without the recourse of a failure criterion, the HFEM is able to predict the correct failure load. More details of this point are given later.

\subsection{Software}

The detailed application of the above micro-macro or meso-macro simulations has been reported in Reference [4]. The post-yielding deformation behavior of ductile sheet metal has also been studied by this heterogeneous finite element method. The major difference is that a commercial implicit-based finite element program was used in Reference [4] with displacement-controlled loading mode. In this work, an in-house explicit finite element program, H3DMAP V7, is used to analyze the burst-pressure tests with load-control loading mode. The hybrid explicit dynamic relaxation solution method is adopted to solve the incremental plasticity problems [9]. The 8-node brick element with reduced integration and von Mises yield criterion are adopted. Pressure is proportionally applied to the inside surface of the finite element model. 


\section{5. $\quad$ Prediction of Failure Pressure}

Both 2-D plane strain and 3-D model simulations have been used to predict the failure pressure. The implicit assumption associated with the use of the 2-D plane strain model is that the crack length is infinite. Figure 4 shows the plane strain and the 3-D finite element models. Two major flaws were considered in the plane strain model. The red line represents indication \#2, and the blue line represents indication \#1. The smallest element size is $0.06 \mathrm{~mm}$ in the plane strain model. Two symmetry planes were utilized in the 3-D model to simplify the simulations. The fixed plane represents the Grayloc hub of the outlet end fitting. In the 3-D model the smallest element size is $0.13 \mathrm{~mm}$, and more than 150,000 elements were used.

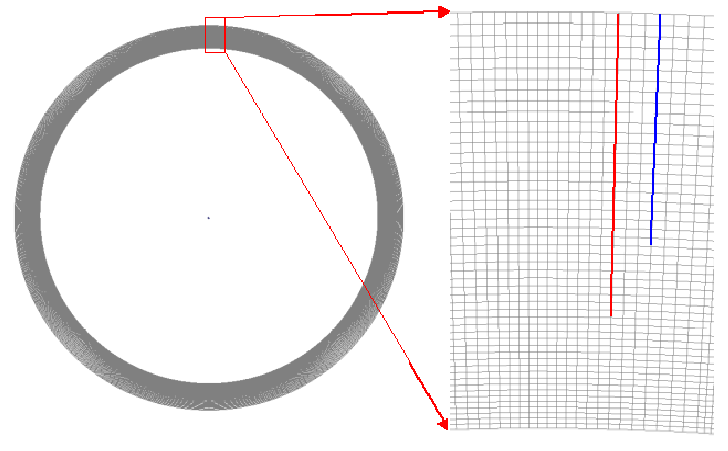

2-D Plane Strain Model

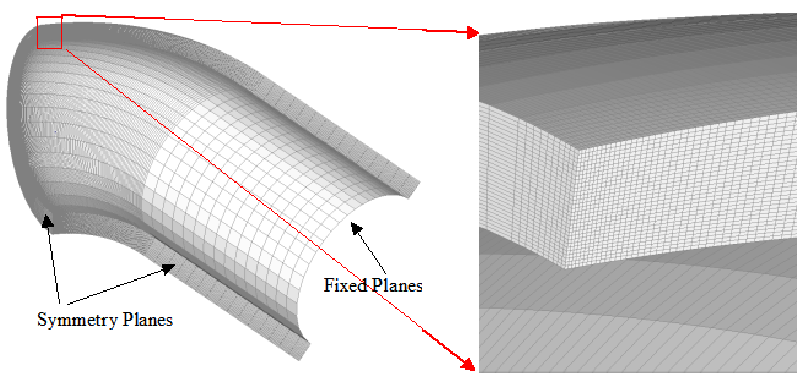

3-D Model

Figure 4: Finite Element Models

For the ductile metals used in the piping industry, substantial local plastic deformation precedes failure in burst-pressure tests. The finite element analysis results from such a process are affected by many factors, i.e., the stress-strain relationship, element formulation, the type of element, mesh size, time step, convergence control, etc. Unlike elastic analysis, the refinement of mesh size in crack analysis does not lead to converged results. In other words, the smaller the mesh size, the greater the strain (or failure parameter) is calculated. It is critical to conduct a sensitivity study on these parameters, and to calibrate the finite element model to a known case before it is applied to similar simulations.

Figure 5 illustrates how the failure pressure is determined using the Rice and Tracey ductile failure criterion. This failure criterion is first calibrated using the simulation of the 2005 burst-pressure test. The grey horizontal lines and thick vertical line represents the measured failure pressure. The intersection between the vertical line and the failure parameter-applied pressure curves represent the critical $C$ values (horizontal lines) as shown in Equation (1). It is clear that the use of different time steps in the simulation results in different $C$ values. The failure pressure for the 2006 test can then be determined using the (2005) calibrated critical $C$ values. Almost the same failure pressure of $53.0 \mathrm{MPa}$ is given by the two simulations (different time step), which agree very well with the actual failure pressure. This agrees with conclusions from Reference [5], i.e., that a relatively small number of time steps can be used to obtain an accurate estimate of the failure load. This observation is useful in that it can be used to obtain significant reductions in the computer time required to perform the simulations. The above simulations for the 2006 test used flaw sizes obtained from the post-test metallurgical examinations. The pre-test simulation, using solely the results of the NDE examinations and engineering insight, predicted a failure pressure of 51.3 MPa, which agrees very well with the actual failure pressure of $51.1 \mathrm{MPa}$.

Figure 6 shows the contour plot of the predicted distribution of the effective plastic strain. It can be seen that the most limiting location is at the maximum depth. Of particular interest is the intense deformation band inclined through the remaining (through-thickness) ligament. Failure of the remaining through-thickness ligament is associated with the development of this band of plasticity, which may also be called a slip-line. Both the 2005 test and the 2006 test show such an inclined failure feature near the inside surface, as circled in Figure 2.

Figure 7 illustrates the determination of failure pressure from the load history plot of effective plastic strain from the 2-D plane strain model. The determined failure pressure is $34.8 \mathrm{MPa}$ for the use of 200,000 time steps. For values of equivalent plastic strain greater than 0.5 , the predicted failure pressure is insensitive to the exact strain value used. In accordance with NB-3213.26, this failure pressure is termed the plastic instability load, which corresponds to "unbounded plastic deformation can occur without an increase in load" [6]. This observation has very important practical implications. It tells us that if the mesh size is sufficiently fine and the time step is sufficiently small, the strain of the element in the failed region increases dramatically with tiny increase in the failure pressure. In other words, the use of the failure criterion like Equation (1) can be avoided for the prediction of failure pressure. It also means that both the element size and the time step are not small enough 
for the present 3-D finite element models to generate a "true" plastic instability load. However, as demonstrated in Figure 5, use of the failure model could significantly reduce the amount of computing resources required.

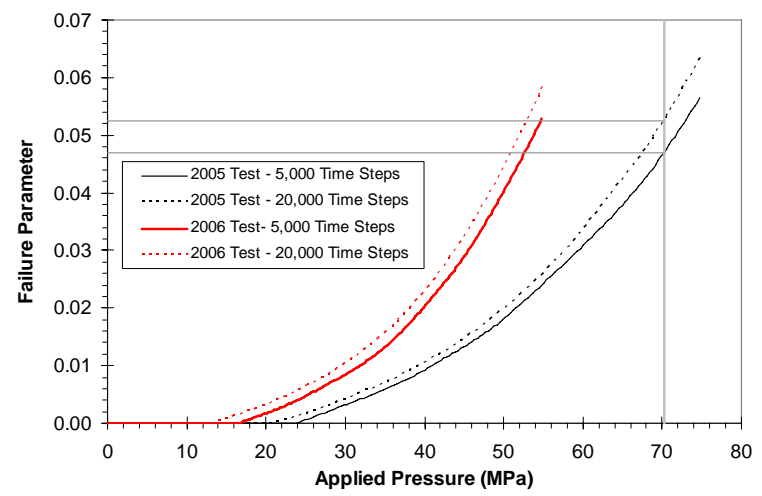

Figure 5: Prediction of Failure Pressure

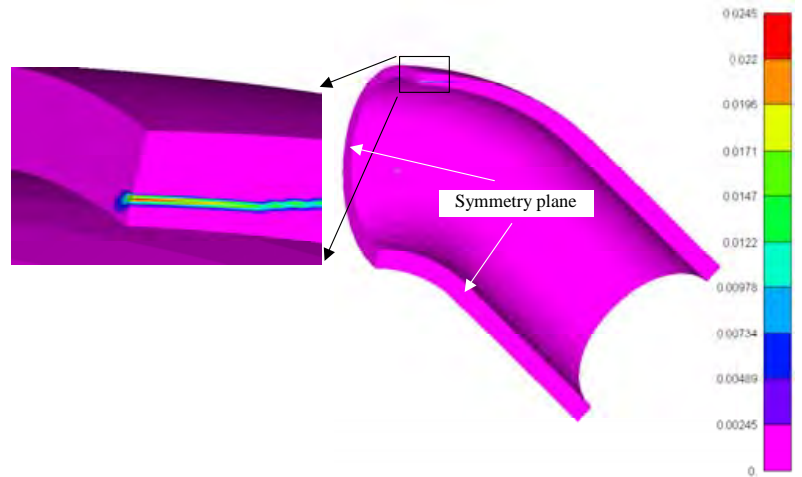

Figure 6: Distribution of Equivalent Plastic Strain

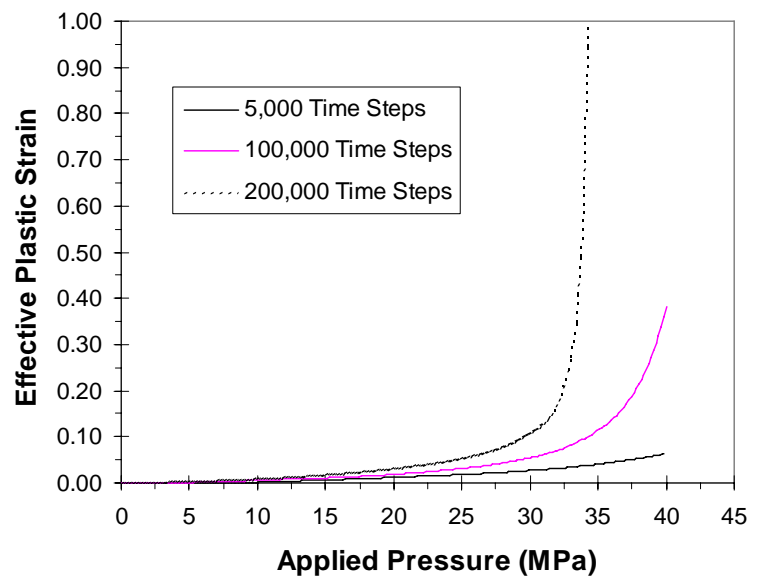

Figure 7: History Plot of Effective Plastic Strain obtained from Plane Strain Model

\section{DISCUSSION}

This section discusses additional studies and assessments that were performed.

\subsection{Consideration of Residual Stress}

The peak residual hoop tensile stress at the outside surface of the extrados of archive tight-radius feeder bends have been measured using the neutron diffraction technique and have values in excess of $200 \mathrm{MPa}$ [11]. This residual stress has been taken into account in previous calculations using FAD assessment methodology. Residual stress is not considered in HFEM because it is significantly lower than the deformation-induced stress values in the vicinity of the crack-tip. Another reason is that the actual residual stress at the crack tip is relaxed during the crack propagation in service. The actual magnitude is believed to be well below $200 \mathrm{MPa}$.

\section{2. $\quad$ Effect of Cold Work on Load Carrying Capacity}

For new and refurbished reactor designs, the residual stresses associated with cold bent feeders are reduced by stress relief heat treatment after the bending of the feeder. Stress relief treatment can reduce residual stress to acceptable levels by 
controlling the stress relief peak temperature. The elongation and fracture toughness are moderately improved, and yield and ultimate strength are also moderately decreased by controlling the length of time for the stress relief heat treatment.

In this section the HFEM (3-D model) is employed to compare the maximum load carrying capacity for the two different materials shown in Figure 3, i.e., assuming the tensile properties for the cold-worked and the parent pipe. The 3-D finite element model shown in Figure 4 is used for this comparison. It has been shown in Sections 3 and 4 that the predicted failure pressure value is $53.0 \mathrm{MPa}$ for cold-worked material. Whereas when the tensile properties for the parent pipe are assumed, the predicted failure pressure value is $39.5 \mathrm{MPa}$. This means that for a given flaw, the cold-worked material has a higher failure load than the parent pipe. This result may appear counter intuitive from the fracture mechanics point of view because of the lower fracture toughness of the cold-worked material. However, the HFEM simulations (as confirmed by the burstpressure tests) show that for these relatively large flaws the failure of the remaining through-wall ligament is associated with plastic instability. The application of traditional fracture mechanics introduces the assumption that the failure is associated with crack extension, and this will result in an under prediction of the failure load in these thin-walled components.

\subsection{Comparison between FAD and HFEM}

It has been mentioned in previous sections that the FAD model of Section XI is not theoretically applicable to coldworked, thin, small size feeder bends. It has been demonstrated that for deep cracks ( $>59 \%$ tw), the failure process is ductile, although the fracture toughness of the cold-worked feeder bend is significantly less than in the parent pipe. Therefore, for the FAD assessment methodology, the failure pressure should be limited by the limit-load cutoff (plastic collapse of the remaining through-wall ligament). The predicted modes of failure are elastic-plastic fracture for D14 and plastic collapse for H12. This would also indicate that the application of plasticity based HFEM to feeder simulations of H12 is appropriate.

The only material input necessary for the HFEM simulation is a single representative stress-strain curve. Fracture toughness is not required. The FAD methodology requires the material characterization for both the tensile and fracture toughness properties of the material. There are no standard test specimens that can be used to characterize the toughness associated with through-wall extension of surface cracks in these thin wall components. Although a 3-D finite element simulation is preferred, it is time consuming. The 2-D plane strain model is much easier to apply, and can provide a conservative lower bound estimate for the plastic instability load. The predicted failure pressures from the 2-D plane strain model are 62.2 $\mathrm{MPa}$ and 34.8 MPa for feeders D14 and H12, respectively. These predictions are much better than predictions obtained using the Section XI FAD procedure.

To predict the failure loads, failure criteria have to be used. FAD methodology uses a bounding curve. Limit load solution is also established as a function of the flaw dimensions and the tensile properties. The HFEM approach adopts the Rice and Tracey ductile failure criterion in the 3-D model. The failure criteria have to be calibrated, usually using the results of a component test. However, as demonstrated in Figure 7, the effective plastic strain criterion does not necessarily be calibrated with respect to previous component test. This is particularly important because it gives the theoretical prediction.

Compared with finite element analyses that use traditional elastic-plastic fracture mechanics, the HFEM methodology has low requirements on the mesh and the element formulations used, which simplify the pre-processing and reduces the analysis time substantially. For an experienced user, a typical 2-D plane strain analysis using the HFEM methodology takes about 1 hour from pre-processing to post-processing. A typical running time for a 3-D model using an average desktop computer (512 MB memory and 3.2 GHz CPU) varies from 1 hour (using 5000 time steps) to 22 hours (using 100,000 time steps).

\section{CONCLUSIONS}

The HFEM methodology has successfully predicted the failure pressure of PLGS feeder H12, which was removed from service because of axial outside surface cracking on the extrados of the second bend. The failure criterion was calibrated using the burst-pressure test results of feeder D14, another ex-service bend with axial surface cracks.

Use of the ductile failure criterion can significantly reduce the computing time without affecting the accuracy of the results as long as the critical failure parameter is carefully calibrated. If sufficiently fine time steps and sufficiently small element size are used, the plastic instability load can be correctly predicted without the recourse to the failure criterion.

Although the cold-worked feeder bends have low fracture toughness, the burst-pressure tests of two ex-service bends with surface axial cracks indicated the failure of the through-wall ligament is a ductile process. For a given flaw, the failure load for the cold-worked material is greater than that for the parent pipe. This is because in these thin-walled components the increase in yield strength associated with the cold-worked material is more significant than the reduced toughness.

\section{ACKNOWLEDGEMENT}

The authors acknowledge the excellent experimental work of Kinectrics for the burst-pressure tests of the two ex-service bends and the measurement of tensile properties. 


\section{REFERENCES}

[1] Gendron, T.S., Slade, J.P., and White, G.A., “The Need for a Probabilistic Approach to Manage Cracking of Carbon Steel Reactor Coolant Piping at the Point Lepreau Generating Station”, OECD/NEA CSNI Workshop Structural Reliability Evaluation and Mechanical Probabilistic Approaches for Examining NPP Components, 2006 September 25-27, Lyon, France.

[2] Slade, J.P., and Gendron, T.S., "Flow Accelerated Corrosion and Cracking of Carbon Steel Piping in Primary Water - Operating Experience at the Point Lepreau Generating Station”, Proceedings of $12^{\text {th }}$ International Conference on Environmental Degradation of Materials in Nuclear Power Systems - Water Reactors, TMS, Salt Lake City, Utah, August 14-18, 2005.

[3] White, G.A, et. al, "Probabilistic Safety Evaluation of Cracking in SA-106 Grade B Carbon Steel- CANDU Reactor Outlet Feeder Piping at Point Lepreau Generating Station”, Proceedings of $6^{\text {th }}$ International Symposium on Contribution of Materials Investigations to Improve the Safety and Performance of LWRs, Fontevraud Royal Abbey, France, September 18-22, 2006.

[4] Duan, X., Jain, M., and Wilkinson, D.S., “Development of a Heterogeneous Microstructurally Based Finite Element Model for the Prediction of Forming Limit Diagram for Sheet Material”, Metallurgical and Materials Transactions A, 2006, Vol. 37A, pp. 3489-3501.

[5] Duan, X., Kozluk, M.J., Pagan, S., and Mills, B.E., "Assessment of the Integrity of Degraded Steam Generator Tube by the Use of Heterogeneous Finite Element Method", Proceedings of the $5^{\text {th }}$ CNS International Steam Generator Conference, Toronto, Ontario, November 26-29, 2006.

[6] The American Society of Mechanical Engineers, “ASME Boiler and Pressure Vessel Code, Section XI”, 2004 Edition, 2004 July.

[7] Zerbst, U., Ainsworth, R.A., and Schwalbe, K.-H., “Basic Principles of Analytical Flaw Assessment Methods”, International Journal of Pressure Vessels and Piping, 2000, Vol. 77, pp. 855-867.

[8] Rice, J.R., and Tracey, D.M., "On the Ductile Enlargement of Voids in Triaxial Stress Fields", Journal of the Mechanics and Physics of Solids, 1969, Vol. 17, pp. 201-217.

[9] Sauvé, R.G., and Metzger, D.R., “A Hybrid Explicit Solution Technique for Quasi-Static Transients”, Proceedings of the 1996 ASME Pressure Vessels and Piping Conference, Montreal, Quebec, Canada, July 21-26,1996, Vol. 326, pp. 151-157.

[10] Budden, P.J., Sharples, J.K., and Dowling, A.R., “The R6 Procedure: Recent Developments and Comparison with Alternative Approaches”, International Journal of Pressure Vessels and Piping, 2000, Vol. 77, pp. 895-903.

[11] Yetisir, M., Donaberger, R., and Rogge, R., "Residual Stresses in CANDU Feeder Bends - Effect of Bend Radius", Proceedings of the 2006 ASME Pressure Vessels and Piping Conference, 2006, Vancouver, B.C., Canada, 2006 July 23-27, Paper Number PVP2006-ICPVT-11-93407. 